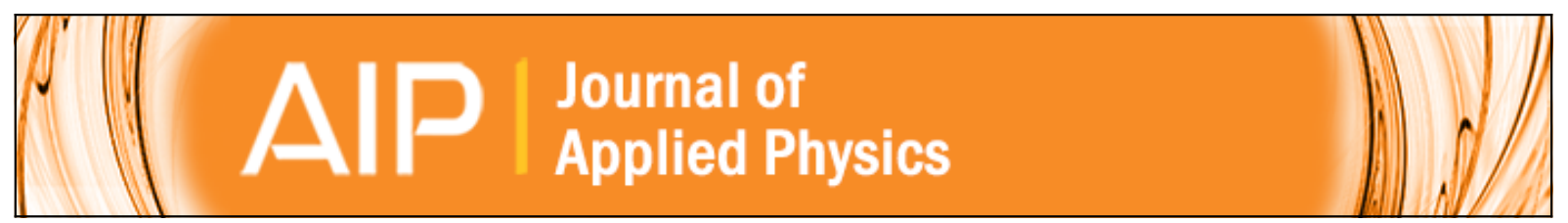

Influence of the annealing atmosphere on the structural properties of FePt thin films

I. A. Vladymyrskyi, M. V. Karpets, F. Ganss, G. L. Katona, D. L. Beke, S. I. Sidorenko, T. Nagata, T. Nabatame,

T. Chikyow, G. Beddies, M. Albrecht, and lu. M. Makogon

Citation: Journal of Applied Physics 114, 164314 (2013); doi: 10.1063/1.4827202

View online: http://dx.doi.org/10.1063/1.4827202

View Table of Contents: http://scitation.aip.org/content/aip/journal/jap/114/16?ver=pdfcov

Published by the AIP Publishing

A AlP Re-register for Table of Content Alerts

Create a profile. 圆 Sign up today! 


\title{
Influence of the annealing atmosphere on the structural properties of FePt thin films
}

\author{
I. A. Vladymyrskyi, ${ }^{1, a)}$ M. V. Karpets, ${ }^{2}$ F. Ganss, ${ }^{3}$ G. L. Katona, ${ }^{4}$ D. L. Beke, ${ }^{4}$ \\ S. I. Sidorenko, ${ }^{1}$ T. Nagata, ${ }^{5}$ T. Nabatame,${ }^{5}$ T. Chikyow, ${ }^{5}$ G. Beddies, ${ }^{3}$ M. Albrecht, ${ }^{3}$ \\ and lu. M. Makogon ${ }^{1}$ \\ ${ }^{1}$ Metal Physics Department, National Technical University of Ukraine "KPI", Prospect Peremogy 37, \\ 03056 Kyiv, Ukraine \\ ${ }^{2}$ I. Frantsevich Institute for Problems of Materials Science, National Academy of Sciences of Ukraine, \\ 3 Krzhizhanovsky Str., 03142 Kyiv, Ukraine \\ ${ }^{3}$ Institute of Physics, Chemnitz University of Technology, Reichenhainer Street 70, 09126 Chemnitz, Germany \\ ${ }^{4}$ Department of Solid State Physics, University of Debrecen, P.O. Box 2, H-4010 Debrecen, Hungary \\ ${ }^{5}$ MANA Foundry and MANA Advanced Device Materials Group, National Institute for Materials Science, \\ 1-1 Namiki, Tsukuba 305-0044, Japan
}

(Received 26 September 2013; accepted 11 October 2013; published online 28 October 2013)

\begin{abstract}
FePt thin films with a thickness of $30 \mathrm{~nm}$ were deposited by dc magnetron sputtering at room temperature onto $\mathrm{SiO}_{2}(100 \mathrm{~nm}) / \mathrm{Si}(100)$ substrates. These films were post-annealed in a temperature range of $500{ }^{\circ} \mathrm{C}$ to $900^{\circ} \mathrm{C}$ for $30 \mathrm{~s}$ in three different atmospheres- $\mathrm{N}_{2}$, Ar, and forming gas $\left(\mathrm{Ar}+\mathrm{H}_{2}\right.$ ( 3 vol. \%)). Irrespective of the annealing atmosphere, the chemically ordered $\mathrm{L} 1_{0} \mathrm{FePt}$ phase has formed after annealing at $500{ }^{\circ} \mathrm{C}$. Higher annealing temperatures in $\mathrm{N}_{2}$ or Ar atmosphere resulted in a strong increase in grain size and surface roughness but also in the appearance of a pronounced (001) texture in the FePt films. However, these films show the presence of iron oxide. In contrast, annealing in forming gas atmosphere suppressed the oxidation process and resulted in a reduced grain size and lower surface roughness. However, no (001)_but a strong (111) — texture was obtained after annealing at $700{ }^{\circ} \mathrm{C}$, which might be related to the reduced unit cell tetragonality and incorporation of hydrogen to the FePt lattice. Thus, this study clearly demonstrates that the oxygen/hydrogen content plays an important role in controlling the crystallographic orientation during post-annealing. (C) 2013 AIP Publishing LLC. [http://dx.doi.org/10.1063/1.4827202]
\end{abstract}

\section{INTRODUCTION}

Chemically ordered $\mathrm{L} 1_{0}$ FePt is a potential material candidate for ultra-high density magnetic data storage due to its large magnetic anisotropy, high saturation magnetization, and high corrosion resistance. ${ }^{1-4}$ Typically, the $\mathrm{L}_{0} \mathrm{FePt}$ phase forms at elevated temperatures when starting from the initially disordered A1 phase. However, industrial application of FePt films requires (i) reduction of the $\mathrm{L} 1_{0}$ phase formation temperature, (ii) inhibition of grain growth, (iii) low surface roughness, and (iv) control of the crystallographic orientation of the grains during heat treatment. Rapid thermal annealing in vacuum is a very promising approach to form $\mathrm{L}_{0}$ ordered $\mathrm{FePt}$ films with pronounced (001) texture, ${ }^{5-10}$ which is induced by thermal tensile stress. ${ }^{11} \mathrm{~A}$ review on recent advances of RTA processed FePt thin films is given in Ref. 12 .

A further approach to modify the ordering kinetics is heat treatment of FePt thin films in inert gases (i.e., $\mathrm{Ar}, \mathrm{N}_{2}$ ) or in forming gases. In this regard, it was shown that nitrogen incorporation forming $\mathrm{FeN}$ enhances the diffusivity of $\mathrm{Fe}$ and $\mathrm{Pt}$ during annealing ${ }^{13-15}$ and thus improves the $\mathrm{L}_{0}$ ordering. In contrast, hydrogen atoms can occupy octahedral interstitials sites in the FePt lattice which causes local strain $^{16}$ influencing the ordering kinetics as well. In addition,

\footnotetext{
a) Author to whom correspondence should be addressed. Electronic mail: vladymyrskyi@kpm.kpi.ua. Tel.: +380444068218.
}

the fast diffusion rate of hydrogen atoms will in turn induce an increased diffusion rate of $\mathrm{Fe}$ and $\mathrm{Pt}$ atoms promoting the L1 $1_{0}$ ordering. ${ }^{16-18}$ Furthermore, hydrogen will suppress oxidation processes. In this regard, Leistner et al. ${ }^{19}$ have shown that annealing of rather thick FePt films in $\mathrm{H}_{2}$ atmosphere leads to better ordering, higher coercivity fields, and smaller averaged grain size as compared to annealing in vacuum. It was also shown that FePt growth at elevated temperatures on $\mathrm{MgO}(100)$ under hydrogen atmosphere leads to smooth and c-axis oriented $\mathrm{L} 1_{0}$ FePt films while under ultra-high vacuum conditions a more granular film morphology was obtained. ${ }^{20}$ Thus, the annealing process in gas atmosphere has a pronounced impact on the structural and related magnetic properties of the FePt films.

In this study, we have investigated the influence of the annealing atmosphere using $\mathrm{N}_{2}$, Ar, and forming gas $\left(\mathrm{Ar}+\mathrm{H}_{2}(3 \mathrm{vol} . \%)\right)$ for annealing temperatures up to $900^{\circ} \mathrm{C}$ on the structural properties of FePt thin films by $\mathrm{x}$-ray diffraction (XRD) and x-ray photoelectron spectroscopy (XPS).

\section{EXPERIMENTAL PROCEDURE}

FePt thin films of $30 \mathrm{~nm}$ thickness were deposited by dc magnetron sputtering at room temperature using individual Fe and Pt targets onto thermally oxidized Si(100) substrates with a 100-nm-thick amorphous $\mathrm{SiO}_{2}$ layer. The Ar sputter pressure was adjusted to $3.5 \times 10^{-3}$ mbar for all depositions and the composition of the $\mathrm{Fe}_{50 \pm 1} \mathrm{Pt}_{50 \pm 1}$ alloy was verified 

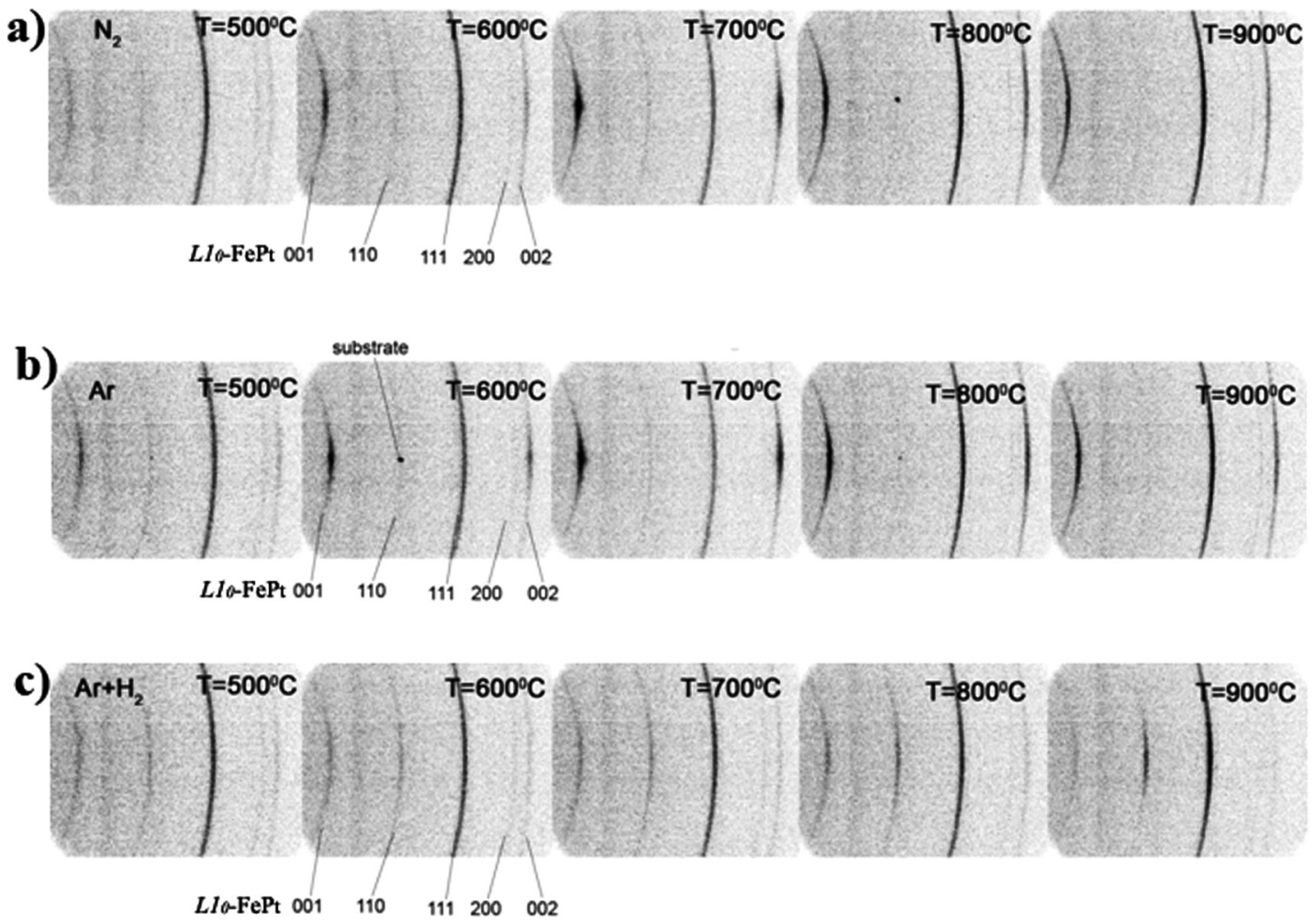

FIG. 1. 2D-XRD images of 30-nm-thick FePt films after annealing at different temperatures in three different atmospheres: (a) $\mathrm{N}_{2}$, (b) $\mathrm{Ar}$, and (c) $\mathrm{Ar}+\mathrm{H}_{2}$ (3 vol. \%).

by Rutherford backscattering spectroscopy. Post-annealing of the film samples up to $900^{\circ} \mathrm{C}$ was carried out in flowing $\mathrm{N}_{2}$, Ar, and forming gas atmosphere at a flowing speed of $0.2 \mathrm{l} / \mathrm{min}$ with annealing times of $30 \mathrm{~s}$ using a fixed heating rate of $10^{\circ} \mathrm{C} / \mathrm{s}$. The thickness of the FePt films was determined by $\mathrm{x}$-ray reflectometry measurements. The structural properties were analyzed using a x-ray diffractometer (Bruker D8 Discover) equipped with a 2-dimensional (2D) detector and with a scintillation counter using $\mathrm{Cu} \mathrm{K}_{\alpha}$ radiation. The average grain size was estimated from XRD data using the Scherrer equation, while the analysis of the film texture was carried out using the March-Dollase model. ${ }^{21}$ In this model, the correction of the intensity of any diffraction peak is performed by introducing an effective repetition factor. In the case, when there is no (001)-texture present, the texture coefficient $(\tau)$ is equal to 1 , and $\tau=0$ when all grains are (001)-oriented, while in intermediate cases $\tau$ takes values between 1 and 0 . Furthermore, XPS was used to analyze the chemical state of Fe and Pt, and the surface roughness was investigated by atomic force microscopy (AFM).

\section{RESULTS AND DISCUSSION}

After post-annealing the FePt films at temperatures up to $900^{\circ} \mathrm{C}$ in different atmospheres, 2D-XRD data were taken. The 2D-XRD patterns are presented in Fig. 1 revealing already after post-annealing at $500{ }^{\circ} \mathrm{C}$ in all three annealing atmospheres the presence of superstructure (001) reflections and the splitting into the tetragonal (200) and (002) peaks confirming that the $\mathrm{L} 1_{0}$ phase has formed. However, it is apparent that after annealing at $700^{\circ} \mathrm{C}$ in $\mathrm{N}_{2}$ and $\mathrm{Ar}$ atmosphere, the
(001) peak intensity becomes much stronger than the intensity of the (111) reflection. Furthermore, the (001) intensity distribution is not uniform along the diffraction ring, which indicates the preferable orientation of the grains along the 001 direction. In contrast, post-annealing in forming gas atmosphere does not lead to the pronounced (001)-texture formation; here, a strong (111)-texture is observed.

Based on the evaluation of the intensity ratio of the (001) and (111) peaks (Fig. 2), the (001)-texture coefficients were extracted as summarized in Fig. 3 as function of annealing temperature for the different gas atmospheres. It shows that grains with preferred (001)-orientation were

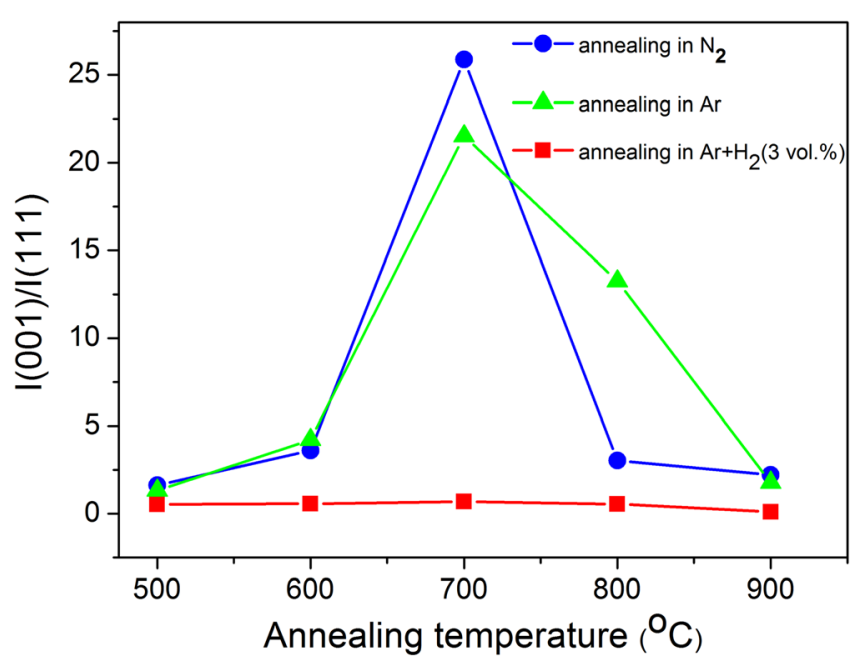

FIG. 2. XRD integral intensity ratio of the (001) and (111) intensity peaks versus annealing temperature in different gas atmospheres. 


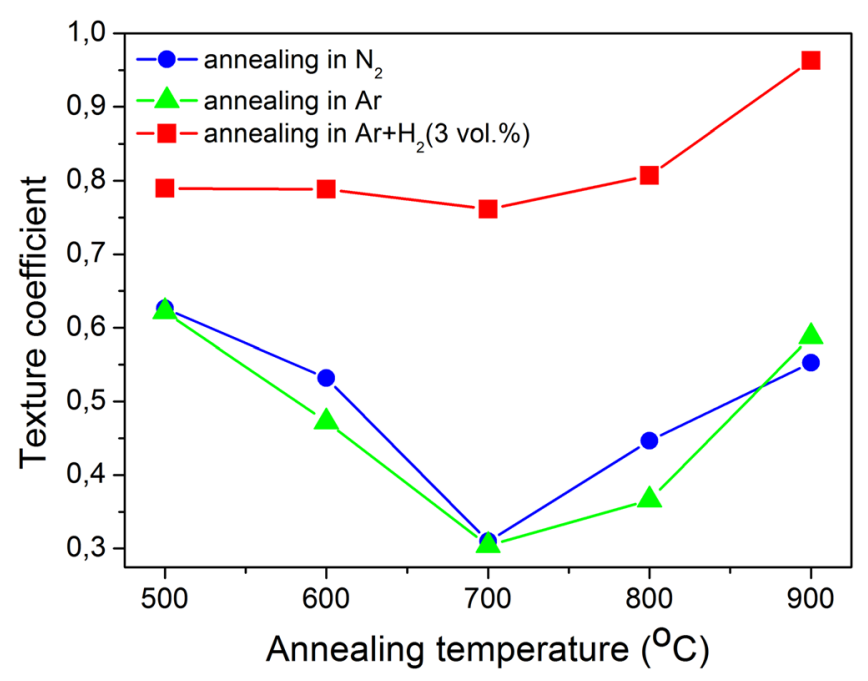

FIG. 3. (001)-texture coefficient of 30-nm-thick FePt films as function of annealing temperature in different gas atmospheres.

formed best after post-annealing in $\mathrm{N}_{2}$ and Ar atmosphere at $700{ }^{\circ} \mathrm{C}$. Further increase of the annealing temperature leads to grain agglomeration combined with the stabilization of the (111)-orientation exhibiting the lowest surface energy. As already mentioned, post-annealing in forming gas atmosphere did not lead to the formation of (001)-texture instead a strong (111)-texture is obtained.

The determination of the a- and c-lattice parameter allows visualizing the strain induced in the grains by the annealing process. As expected the c-axis is substantially contracted, while the a-axis is expanded in agreement with the bulk values of fully $\mathrm{L}_{0}$ ordered FePt resulting in a tetragonal distortion as presented by the c/a values shown in Fig. 4. Here, the films annealed in forming gas reveal a somewhat smaller tetragonal distortion most likely due to the incorporation of hydrogen to the FePt lattice.

The average grain size as a function of the annealing temperature is shown in Fig. 5. Annealing of films at temperatures higher than $700{ }^{\circ} \mathrm{C}$ in $\mathrm{N}_{2}$ or $\mathrm{Ar}$ atmosphere leads to a significant increase in grain size due to grain agglomeration

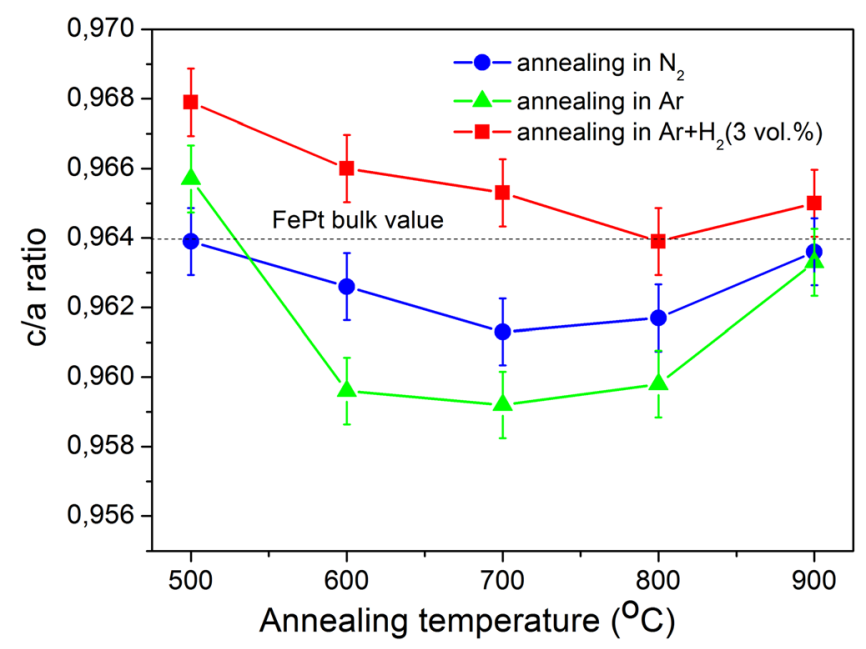

FIG. 4. Tetragonality (c/a ratio) of 30-nm-thick FePt films as function of annealing temperature in different gas atmospheres. The bulk c/a ratio of fully $\mathrm{L} 1_{0}$ ordered FePt is marked by a dotted line.

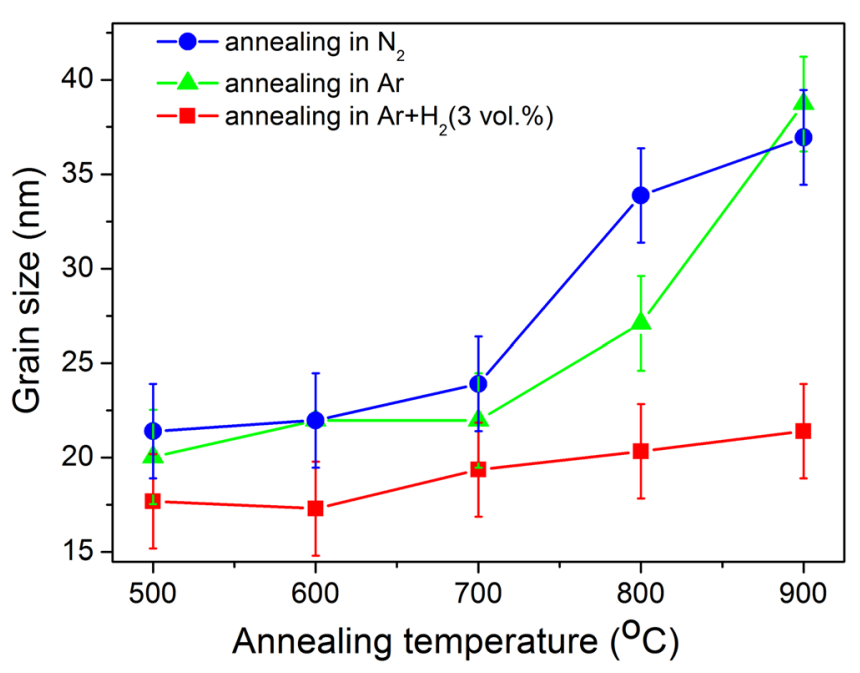

FIG. 5. Averaged grain size of 30-nm-thick FePt films as function of annealing temperature in different gas atmospheres.

resulting in the stabilization of the (111) orientation. This grain growth process is strongly suppressed when annealed in forming gas showing an averaged grain size of about $20 \mathrm{~nm}$ at $900{ }^{\circ} \mathrm{C}$, which is two times smaller than compared with annealing in $\mathrm{N}_{2}$ and $\mathrm{Ar}$ atmosphere. In turn, this leads also to a reduced root mean square (rms) surface roughness as extracted from AFM imaging (Fig. 6).

In order to get some insight on the influence of hydrogen in particular on the oxidation process of the film samples during annealing, XPS spectra were taken around the Fe $2 p$ and $\mathrm{Pt} 4 \mathrm{f}$ peaks. Fig. 7 shows the Fe 2p and Pt 4f XPS spectra of FePt films after post-annealing in different atmospheres at $800{ }^{\circ} \mathrm{C}$. After annealing in $\mathrm{H}_{2}$-containing atmosphere, the Fe 2 p peak appears at about $707.4 \mathrm{eV}$, which corresponds to the bulk value for pure iron. ${ }^{22}$ After annealing in $\mathrm{N}_{2}$ and $\mathrm{Ar}$ atmosphere, this peak shifts to $711.3 \mathrm{eV}$ indicating the presence of iron oxide (i.e., $\mathrm{Fe}_{2} \mathrm{O}_{3}$ ). ${ }^{22}$ The $\mathrm{Pt} 4 \mathrm{f}$ peak positions are close to their expected bulk value known from literature ${ }^{13,22}$ and only a small shift of $0.6 \mathrm{eV}$ is observed for the sample post annealed in $\mathrm{N}_{2}$.

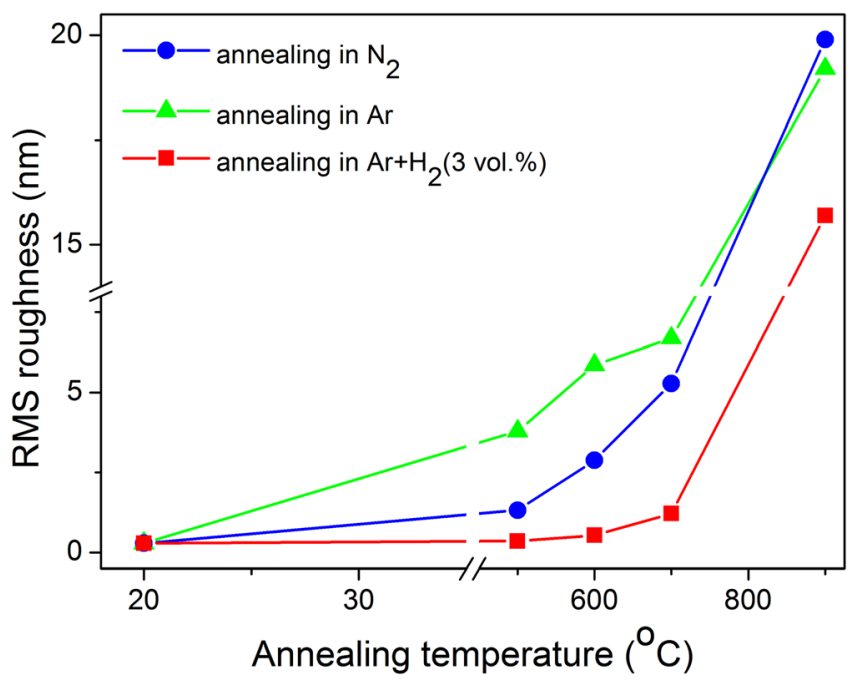

FIG. 6. RMS roughness of 30-nm-thick FePt films as function of annealing temperature in different gas atmospheres. 

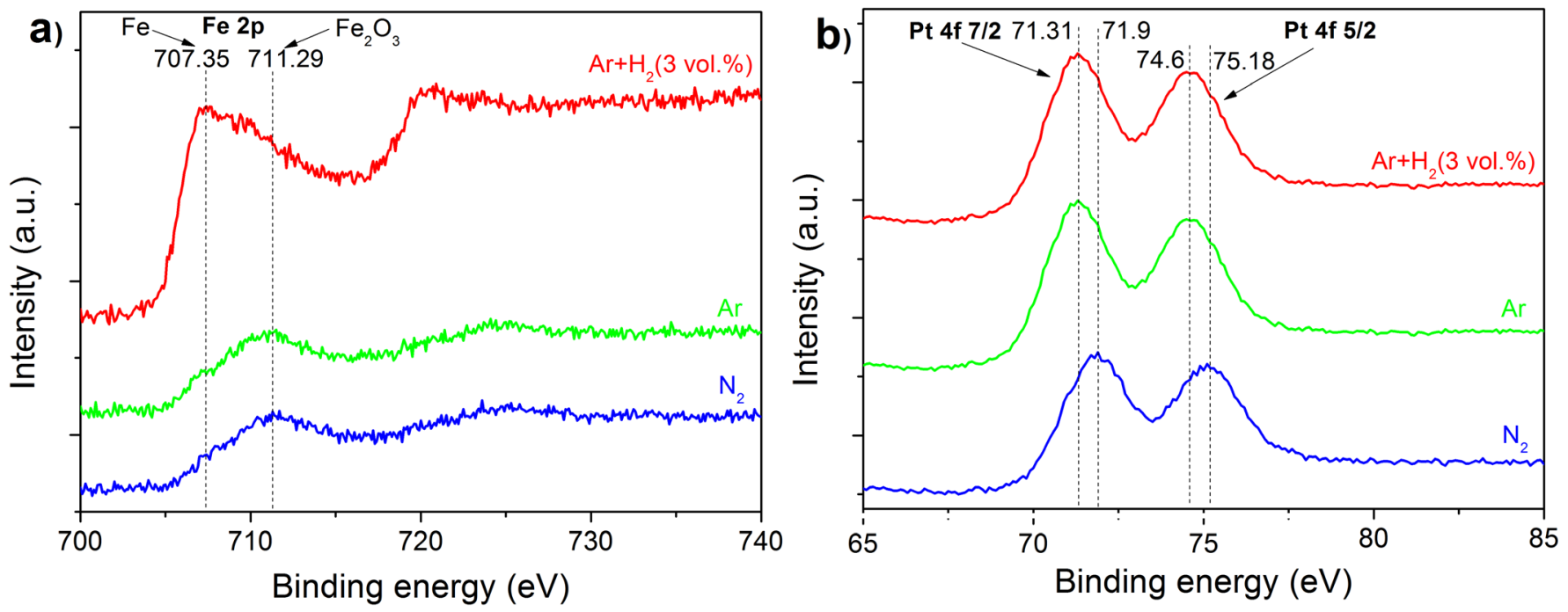

FIG. 7. (a) Fe 2p and (b) Pt 4f XPS spectra of 30-nm-thick FePt films after annealing at $800^{\circ} \mathrm{C}$ in three different atmospheres.

The observed differences in the structural properties of FePt films after post-annealing in different atmospheres can be explained by the influence of oxygen and hydrogen. The larger tetragonality in FePt after annealing in $\mathrm{Ar}$ and $\mathrm{N}_{2}$ atmosphere might indicate the penetration of oxygen (which is present in inert gases and in the residual atmosphere) into the film and the formation of $\mathrm{Fe}$ oxides as confirmed by XPS investigations. This distortion adds elastic stress to the system which is the origin of tensile in-plane strain favoring the growth of (001)-oriented grains with the longer axis of the fct lattice pointing in the direction of the strain. ${ }^{11}$ In contrast, the presence of hydrogen drastically reduces the amount of oxygen in the gas atmosphere. Furthermore, hydrogen is incorporated into the FePt lattice and reduces the tetragonality of the $\mathrm{L} 1_{0}$ unit cell thus the driving force for (001) texture formation is slightly reduced. Moreover, hydrogen promotes the kinetics of ordering and thus lowers the ordering temperature. ${ }^{19}$ Both effects, the simultaneous reduction of oxygen and the incorporation of hydrogen to the FePt lattice, are the key ingredients for the (111) texture formation as also pointed out in Ref. 23.

\section{CONCLUSIONS}

We can conclude that annealing in $\mathrm{N}_{2}$ and Ar atmosphere at $700^{\circ} \mathrm{C}$ leads to a pronounced (001) texture formation of the $\mathrm{L}_{0}$ ordered $\mathrm{FePt}$ films. In addition, a strong oxidation of the film samples as evidenced by XPS investigations was detected. Furthermore, for these films a significant increase in grain size and surface roughness was observed. In contrast, annealing in forming gas atmosphere suppressed the oxidation process and resulted in a reduced grain size and lower surface roughness. However, no (001)-but a strong (111)-texture was obtained after annealing at $700{ }^{\circ} \mathrm{C}$, which might be related to the slightly reduced unit cell tetragonality and incorporation of hydrogen to the FePt lattice. Thus, this study clearly demonstrates that the oxygen/hydrogen content plays an important role in controlling the crystallographic orientation during post-annealing.

\section{ACKNOWLEDGMENTS}

This work was financially supported by the German Academic Exchange Service (DAAD) in the frame of Leonard Euler scholarship program (Grant ID 55576194). The project was also funded by OTKA (Grant No. 80126) and by the TAMOP-4.2.2.A-11/1/KONV-2012-0036 project, which is part of the New Hungary Development Plan co-financed by the European Social Fund and the European Regional Development Fund. This research was also supported by the European Union and the State of Hungary, co-financed by the European Social Fund in the framework of TÁMOP 4.2.4. A/2-11-1-2012-0001 "National Excellence Program" (author G. L. Katona). Support from the Hungarian Chinese bilateral project, TÉT_12_CN-1-2012-0036, is also acknowledged.

${ }^{1}$ D. Weller, O. Mosendz, G. Parker, S. Pisana, and T. S. Santos, Phys. Status Solidi A 210, 1245 (2013).

${ }^{2}$ J. Lyubina, B. Rellinghaus, O. Gutfleisch, and M. Albrecht, "Structure and magnetic properties of $L 1_{0}$-ordered Fe-Pt alloys and nanoparticles," in Handbook of Magnetic Materials, edited by K. H. J. Buschow (Elsevier, 2011), Vol. 19, pp. 291-395.

${ }^{3}$ Y. Shiroishi, K. Fukuda, I. Tagawa, H. Iwasaki, S. Takenoiri, H. Tanaka, H. Mutoh, and N. Yoshikawa, IEEE Trans. Magn. 45, 3816 (2009).

${ }^{4}$ Development in Data Storage, Materials Perspective, edited by S. N. Piramanayagam and T. C. Chong (John Wiley \& Sons, Inc., New York, 2012).

${ }^{5}$ P. Rasmussen, X. Rui, and J. E. Shield, Appl. Phys. Lett. 86, 191915 (2005).

${ }^{6}$ T. Narisawa, T. Hasegawa, S. Ishio, and Y. Yamane, J. Appl. Phys. 109, 033918 (2011).

${ }^{7}$ S. N. Hsiao, S. H. Liu, S. K. Chen, T. S. Chin, and H. Y. Lee, Appl. Phys. Lett. 100, 261909 (2012).

${ }^{8}$ C. Brombacher, C. Schubert, K. Neupert, M. Kehr, J. Donges, and M. Albrecht, J. Phys. D, Appl. Phys. 44, 355001 (2011).

${ }^{9}$ J. S. Kim, Y. M. Koo, and N. Shin, J. Appl. Phys. 100, 093909 (2006).

${ }^{10}$ S. N. Hsiao, F. T. Yuan, H. W. Chang, H. W. Huang, S. K. Chen, and H. Y. Lee, Appl. Phys. Lett. 94, 232505 (2009).

${ }^{11}$ L.-W. Wang, W.-C. Shih, Y.-C. Wu, and C.-H. Lai, Appl. Phys. Lett. 101, 252403 (2012).

${ }^{12}$ M. Albrecht and C. Brombacher, Phys. Status Solidi A 210, 1272-1281 (2013).

${ }^{13}$ W. B. Mi, E. Y. Jiang, and H. L. Bai, J. Appl. Phys. 99, 034315 (2006).

${ }^{14}$ V. Phatak, A. Gupta, V. R. Reddy, S. Chakravarty, H. Schmidt, and R. Rüffer, Acta Mater. 58, 979 (2010). 
${ }^{15}$ Y. Tran and C. D. Wright, J. Magn. Magn. Mater. 331, 216 (2013).

${ }^{16}$ C. H. Lai, Y. C. Wu, and C. C. Chiang, J. Appl. Phys. 97, $10 \mathrm{H} 305$ (2005).

${ }^{17}$ K. R. Coffey, M. A. Parker, and J. K. Howard, IEEE Trans. Magn. 31, 2737 (1995).

${ }^{18}$ T. S. Vedantam, J. P. Liu, H. Zeng, and S. Sun, J. Appl. Phys. 93, 7184 (2003).

${ }^{19}$ K. Leistner, J. Thomas, H. Schlörb, M. Weisheit, L. Schultz, and S. Fähler, Appl. Phys. Lett. 85, 3498 (2004).
${ }^{20}$ R. Maaß, M. Weisheit, S. Fähler, and L. Schultz, J. Appl. Phys. 100, 073910 (2006).

${ }^{21}$ W. A. Dollase, J. Appl. Crystallogr. 19, 267-272 (1986).

${ }^{22}$ J. F. Moulder and J. Chastain, Handbook of X-Ray Photoelectron Spectroscopy: A Reference Book of Standard Spectra for Identification and Interpretation of XPS Data (Physical Electronics Division, PerkinElmer Corp, Eden Prairie, Minnesota, 1992).

${ }^{23}$ J. S. Chen, B. C. Lim, and J. P. Wang, Appl. Phys. Lett. 81, 1848 (2002). 